\title{
MicroRNA-181a enhances the chemotherapeutic sensitivity of chronic myeloid leukemia to imatinib
}

\author{
GUANGYU WANG $^{1 *}$, RAN ZHAO $^{2 *}$, XINGSHENG ZHAO $^{3 *}$, XI CHEN $^{4}$, DONG WANG $^{5}$, YINJI JIN ${ }^{2}$,

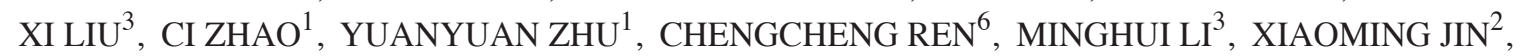 \\ FENGMIN ZHANG ${ }^{7}$, ZHAOHUA ZHONG $^{7}$, TIANZHEN WANG ${ }^{2}$ and XIAOBO $\mathrm{LI}^{2,8,9}$
}

\author{
${ }^{1}$ Department of Gastrointestinal Medical Oncology, The Affiliated Tumor Hospital of Harbin Medical University, Harbin, \\ Heilongjiang 150081; ${ }^{2}$ Department of Pathology, Harbin Medical University, Harbin, Heilongjiang 150086; \\ ${ }^{3}$ Department of Cardiovascular, Inner Mongolia People's Hospital, Hohhot, Inner Mongolia 010070; \\ ${ }^{4}$ Department of Hematology, The Second Affiliated Hospital, Harbin Medical University, Harbin, Heilongjiang 150086; \\ ${ }^{5}$ Department of Oncology and Hematology, Inner Mongolia Tongliao City Hospital, Tongliao, Inner Mongolia 028000; \\ ${ }^{6}$ Department of Obstetrics and Gynecology, The First Affiliated Hospital, Harbin Medical University, Harbin, \\ Heilongjiang 150001; ${ }^{7}$ Department of Microbiology, Harbin Medical University, \\ Harbin, Heilongjiang 150081; ${ }^{8}$ Translational Medicine Center of Northern China, Harbin Medical University; \\ ${ }^{9}$ Basic Medical Institute, Heilongjiang Medical Science Academy, Harbin, Heilongjiang 150086, P.R. China
}

Received October 22, 2014; Accepted August 17, 2015

DOI: $10.3892 / 01.2015 .3663$

\begin{abstract}
MicroRNA-181 (miR-181) has been recently demonstrated to participate in the differentiation and development of immune cells, including natural killer cells and B and $\mathrm{T}$ lymphocytes, and myeloid linages, including erythroid and megakaryocytic cells. The aberrant expression of miR-181, particularly low expression levels, has been observed in a number of leukemia types, including B-cell chronic lymphocytic leukemia and cytogenetically abnormal acute myeloid leukemia. However, the expression and function of miR-181 in chronic myeloid leukemia (CML) remains unknown. In the present study, the aberrant expression of miR-181a was analyzed in a patient with CML and in the CML K562 cell line. In addition, the function and potential mechanisms of miR-181a in K562 cells with regard to their chemotherapeutic sensitivity to imatinib were investigated. The expression levels of miR-181a were significantly reduced in the patient with CML and in the CML K562 cell line. Furthermore, the overexpression of miR-181a in the K562 cells enhanced the chemotherapeutic sensitivity of these cells to imatinib. The
\end{abstract}

Correspondence to: $\mathrm{Dr}$ Xiaobo $\mathrm{Li}$ and Dr Tianzhen Wang, Department of Pathology, Harbin Medical University, 194 Xufu Road, Harbin, Heilongjiang 150086, P.R. China

E-mail: lixiaobo@ems.hrbmu.edu.cn

E-mail: wtzpath@163.com

${ }^{*}$ Contributed equally

Key words: microRNA-181a, chemotherapeutic sensitivity, chronic myeloid leukemia potential mechanism mediating these effects may be associated with the capacity of miR-181a to inhibit cell growth and/or to induce cells apoptosis and differentiation in K562 cells. The results of the present study suggested that miR-181a may be a target for the treatment of CML and a useful indicator of the therapeutic sensitivity of CML to imatinib.

\section{Introduction}

MicroRNAs (miRNAs/miRs) are a novel class of regulatory molecules that function mainly at the post-transcriptional level by modulating the expression of their target genes. miRNAs are generated by stepwise cleavage of their genome-encoded transcripts via the RNase W enzymes Drosha and Dicer. In this process, one strand of the double-stranded-RNA cleavage products is incorporated into the active ribonucleoprotein complex known as the RNA-induced silencing complex, which results in the degradation of the target mRNA, and/or the repression of its translation $(1,2)$.

Previous studies have reported that miRNAs are involved in hematopoiesis and leukemogenesis. Chen et al (3) first identified the preferential expression of miR-181 in B-lymphoid cells of the bone marrow in mice. The study observed that the ectopic expression of miR-181 in hematopoietic stem/progenitor cells led to an increase of B-lineage cells in tissue-culture differentiation assays and in adult mice (3). Previous studies demonstrated that miR-181a promoted the ontogenesis, differentiation and development of natural killer cells, and was also critical for the development and selection of T lymphocytes (4-8). Choong et al (9) noted that the expression of miR-181a was upregulated during the primary stage of erythropoiesis in umbilical cord blood-derived cluster of differentiation (CD) $34^{+}$cells. A recent study by Li et al (10) demonstrated that miR-181 acted as a critical molecular switch 
for megakaryocytic hematopoiesis by interrupting the Lin28 and let-7 feedback circuit. Additionally, Pekarsky et al (11) identified the aberrant expression of miR-181 in leukemia. In the study, it was observed that the expression levels of miR-181 in B-cell chronic lymphocytic leukemia (B-CLL) were inversely correlated with those of Tcl1, and that the expression of Tcl1 was regulated by miR-181. Calin et al (12) further demonstrated that the high expression levels of the oncogene Tcl1 in patients with B-CLL were due to the low expression levels of miR-181 and miR-29 detected in these patients, which resulted in the pathogenesis of the aggressive form of B-CLL displayed by these patients. Previous studies reported that the upregulation of the miR-181 family was one of the high-risk molecular factors involved in the development of cytogenetically normal acute myeloid leukemia (CN-AML), while the downregulation of miR-181 was associated with an adverse prognosis in patients with cytogenetically abnormal AML $(13,14)$.

To date, the role and aberrant expression pattern of miR-181 in CML remain unknown. In the present study, the potential alterations in the expression pattern of miR-181a were evaluated in a patient with CML and in the CML K562 cell line. Furthermore, the function and potential mechanism of miR-181a in mediating the chemotherapeutic sensitivity of K562 cells to the therapeutic drug imatinib were also investigated.

\section{Materials and methods}

Blood samples. A patient with CML, diagnosed at The Second Affiliated Hospital of Harbin Medical University (Harbin, Heilongjiang, China), and 3 healthy volunteers, who were used as controls, were recruited for the present study. Once informed consent had been obtained from all the participants in the study, whole blood was collected, and mononuclear cells were isolated by density gradient with Percoll (density, 1.077 g/ml; Amersham Pharmacia Biotech Europe GmbH, Munich, Germany), according to the manufacturer's instructions. The present study was approved by the Ethics Committee of Harbin Medical University.

Cell culture. The CML K562 cell line was acquired from the Institute of Basic Medical Science (Chinese Academy of Medical Science, Beijing, China), while the 293T/17 cell line used for lentivirus packaging was purchased from the American Type Culture Collection (Manassas, VA, USA). The cells were cultured in Dulbecco's modified Eagle's medium (DMEM) (Gibco Life Technologies, Beijing, China) containing 10\% (v/v) fetal bovine serum, penicillin $(100 \mathrm{U} / \mathrm{ml})$ and streptomycin $(100 \mu \mathrm{g} / \mathrm{ml})$, and incubated at $37^{\circ} \mathrm{C}$ in the presence of $5 \% \mathrm{CO}_{2}$.

miR-181a overexpression, lentiviral vector construction, virus packaging and cell infection. The hsa-miR-181a gene was amplified from the genomic DNA of normal human leukocytes by polymerase chain reaction (PCR), using the primers listed in Table I. The amplified hsa-miR-181a gene was sequenced by Genewiz company (Beijing, China) and digested with BamHI (New England Biolabs, Inc., Ipswich, MA, USA, prior to be cloned into the pC-1 plasmid (Addgene, Inc., Cambridge, MA, USA), which was linearized as previously described (10). Next, the lentiviral vectors were packaged with a lentiviral vector packaging kit (System Biosciences, Mountain View, CA, USA), according to the manufacturer's instructions. K562 cells $\left(1 \times 10^{5}\right)$ were plated into each well of a 24 -well plate, and $10 \mu 1$ of $1 \times 10^{8}$ $\mathrm{IU} / \mathrm{ml} \mathrm{miR}-181 \mathrm{a}$ and enhanced green fluorescent protein (EGFP) lentiviral vectors were added into 24 -well plate and incubated for $12 \mathrm{~h}$, respectively. Subsequently, lentiviral vector-infected K562 cells were transferred to a $25 \mathrm{~cm}^{2}$ flask and grown for at least $72 \mathrm{~h}$ prior to be sorted using a FACSAria II cell sorter (BD Biosciences, Franklin Lakes, NJ, USA). The purified lentiviral vectors infected K562 cells were denoted as K562-miR-181a and K562-EGFP, respectively.

$R N A$ isolation and reverse transcription-quantitative $P C R$ $(R T-q P C R)$. Total RNA was extracted from the harvested cells using TRIzol (Invitrogen Life Technologies, Carlsbad, CA, USA), and quantified using the absorbance at $260 \mathrm{~nm}$. cDNA was synthesized from $2 \mu \mathrm{g}$ of total RNA with M-MLV reverse transcriptase (Invitrogen Life Technologies). Oligo(dT) $)_{18}$ and stem-loop primers were used as primers for the RT of mRNAs and miRNAs, respectively. RT-qPCR was performed with a Bio-Rad Real-Time PCR System (Bio-Rad Laboratories, Inc., Hercules, CA, USA) using a SYBR Premix Ex Taq kit (Takara Biotechnology Co., Ltd., Dalian, China), according to the manufacturer's instructions. The relative expression levels of the genes of interest were analyzed by the $2^{-\Delta \Delta \mathrm{Ct}}$ method. GAPDH and U6 were used as endogenous controls for the quantification of mRNA and miRNA, respectively. The sequence of the primers used for the RT of miR-181a and U6, and for the qPCR analysis, are summarized in Table I.

Cell viability assay. Cell viability was measured using a CellTiter-Glo Luminescent Cell Viability Assay kit (Promega Corporation, Madison, WI, USA) according to the manufacturer's instructions. For the assay, $1 \times 10^{4}$ K562-miR-181a and the control K562-EGFP cells, which had been treated with DMEM containing 0.05, 0.1, 0.2 or $0.4 \mu \mathrm{M}$ imatinib (Selleck Chemicals, Houston, TX, USA) for $72 \mathrm{~h}$ prior to the assay, were employed.

Cell apoptosis assay. For the apoptosis assay, $5 \times 10^{5}$ K562-miR-181a and K562-EGFP cells were exposed to $0.3 \mu \mathrm{M}$ imatinib for $24 \mathrm{~h}$, and subsequently, cell apoptosis was detected with an apoptosis kit (BD Biosciences). The cells were collected, washed and resuspended in binding buffer at a density of $1 \times 10^{6}$ cells $/ \mathrm{ml}$. Next, $1 \times 10^{5}$ cells were incubated for 15 min with $5 \mu$ l phycoerythrin (PE)-Annexin V and $5 \mu 17$-aminoactinomycin $\mathrm{D}$ at room temperature in the dark, and analyzed using a FACSCanto II flow cytometer (BD Biosciences) $1 \mathrm{~h}$ later.

Cell proliferation assay. For the cell proliferation assay, $2 \times 10^{5}$ cells were cultured in a $10-\mathrm{cm}$ dish and incubated in complete medium at $37^{\circ} \mathrm{C}$ in the presence of $5 \% \mathrm{CO}_{2}$. The cells were counted after different time intervals using a cell counting chamber, and the cell growth curve was represented according to the logarithmic value of the number of cells measured at the different time-points.

Cell cycle analysis. To analyze the cell cycle, the K562 cells were harvested, washed twice with phosphate-buffered saline 
Table I. Primers used in the present study.

\begin{tabular}{|c|c|}
\hline Primer & Sequence $\left(5^{\prime}-3^{\prime}\right)$ \\
\hline \multicolumn{2}{|c|}{ miR-181a PCR amplification } \\
\hline Forward & GGATCCTGTTGTTTCTGTCTCCCATC \\
\hline Reverse & GGATCCCACCGACAGCAATG \\
\hline miR-181a RT & GTCGTATCCAGTGCAGGGTCCGAGGTATTCGCACTGGATACGACACGTGG \\
\hline U6 RT & AAAATATGGAACGCTTCACGAATTTG \\
\hline \multicolumn{2}{|l|}{ miR-181a } \\
\hline Forward & CACATGACTGTTATCGCCATCTACT \\
\hline Reverse & GTCGTATCCAGTGCAGGGTCCGAGGT \\
\hline \multicolumn{2}{|l|}{ U6 } \\
\hline Forward & CTCGCTTCGGCAGCACATATACT \\
\hline Reverse & ACGCTTCACGAATTTGCGTGTC \\
\hline \multicolumn{2}{|l|}{ GAPDH } \\
\hline Forward & ATGGGGAAGGTGAAGGTCG \\
\hline Reverse & GGGGTCATTGATGGCAACAATA \\
\hline \multicolumn{2}{|l|}{$\gamma$-globin } \\
\hline Forward & GCAGCTTGTCACAGTGCAGTTC \\
\hline Reverse & TGGCAAGAAGGTGCTGACTTC \\
\hline \multicolumn{2}{|l|}{ CD235A } \\
\hline Forward & GGCTGGTGTTATTGGAACGATC \\
\hline Reverse & GAGGTTTTACATCAGATGGGCTTT \\
\hline \multicolumn{2}{|l|}{ CD41 } \\
\hline Forward & GATGAGACCCGAAATGTAGGC \\
\hline Reverse & TCAGTCTTTTCTAGGACGTTCCA \\
\hline \multicolumn{2}{|l|}{ CD61 } \\
\hline Forward & AGGATGACTGTGTCGTCAGAT \\
\hline Reverse & GGTAGACGTGGCCTCTTTATACA \\
\hline
\end{tabular}

miR-181a, microRNA-181a; PCR, polymerase chain reaction; RT, reverse transcription; CD, cluster of differentiation.

(PBS), and fixed in $75 \%$ ethanol at $4^{\circ} \mathrm{C}$ overnight. Following 2 washes with ice-cold PBS, the cells were incubated with RNaseA $(20 \mu \mathrm{g} / \mathrm{ml})$ at $37^{\circ} \mathrm{C}$ for $30 \mathrm{~min}$, and stained with propidium iodide (PI) $(0.5 \mathrm{mg} / \mathrm{ml})$ at $4^{\circ} \mathrm{C}$ for $30 \mathrm{~min}$. The cells were then washed with PBS containing $1 \%$ bovine serum albumin, and resuspended in $500 \mu \mathrm{l}$ PBS. Flow cytometric data of PI were acquired from $\sim 10^{5}$ cells using a flow cytometer.

Induction and determination of $K 562$ cell differentiation. The K562 cells were seeded at a density of $5 \times 10^{5}$ cells $/ \mathrm{ml}$ in DMEM, supplemented with $50 \mu \mathrm{M}$ hemin (Sigma-Aldrich, St. Louis, USA) to induce erythroid differentiation or $25 \mu \mathrm{M}$ TPA (Sigma-Aldrich) for megakaryocytic differentiation. The erythroid differentiation of K562 cells were analyzed based on the expression of $\gamma$-globin and CD235a using RT-qPCR or $20 \mu \mathrm{g} / \mathrm{ml}$ benzidine staining (Sigma-Aldrich). The megakaryocytic differentiation of K562 cells was measured by determining the expression of CD41 and CD61 using RT-qPCR. Primers used for RT-qPCR are listed in Table I.

Statistical analysis. Data were presented as the mean \pm standard deviation and subjected to a one-way analysis of variance
(ANOVA). A Student's t-test was used to compare the relative expression of target genes and the ANOVA analysis was used to test the effect of miR-181a on the chemotherapeutic sensitivity of K562 cells to imatinib. $\mathrm{P}<0.05$ was considered to indicate statistically significance. SPSS software version 10.0 for windows (SPSS, Inc., Chicago, IL, USA) was used to conduct the statistical analyses.

\section{Results}

Reduced expression of miR-181a in the CML patient and K562 cell line. In order to detect potential alterations in the expression pattern of miR-181a in CML, the expression levels of miR-181a were measured by RT-qPCR in 1 patient with CML and in the CML K562 cell line, and compared with the expression levels of miR-181a in 3 healthy volunteers used as controls. The results indicated that the expression levels of miR-181a were significantly reduced in the patient with CML and in the CML K562 cells (Fig. 1).

Overexpression of miR-181a enhances the chemotherapy sensitivity of $K 562$ cells to imatinib. To investigate the potential 


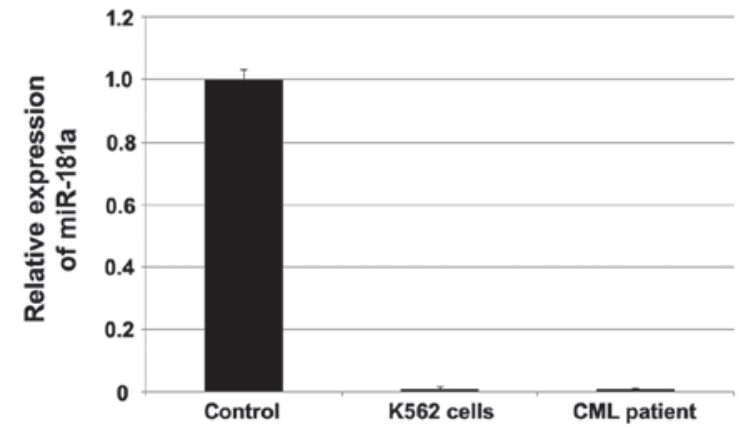

Figure 1. Aberrant expression of miR-181a in CML. Reverse transcription-quantitative polymerase chain reaction analysis revealed lower expression levels of miR-181a in 1 patient with CML and in CML K562 cells, compared with the healthy volunteer control group $(\mathrm{P}<0.01)$. The results are presented as the mean \pm standard deviation from two experiments. miR-181a, microRNA-181a; CML, chronic myeloid leukemia.

A

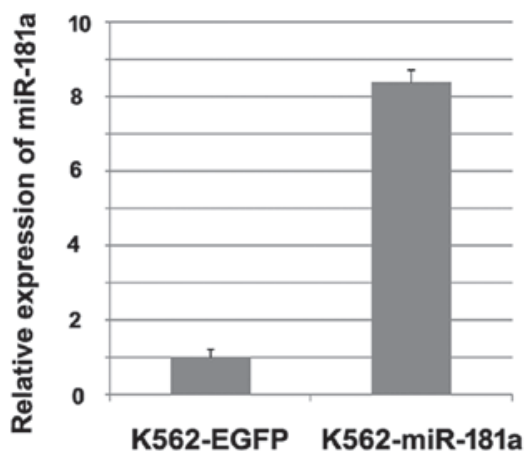

B

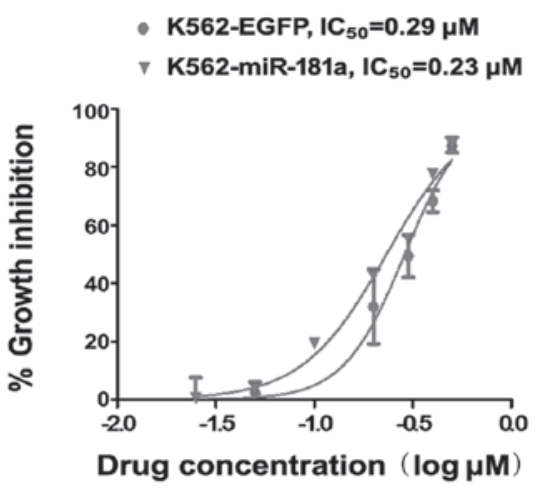

Figure 2. Overexpression of miR-181a in chronic myeloid leukemia K562 cells enhanced the chemotherapeutic sensitivity of these cells to the drug imatinib. (A) The overexpression of miR-181a in K562 cells infected with a lentivirus carrying the miR-181a gene was confirmed by reverse transcription-quantitative polymerase chain reaction analysis. K562 cells infected with a lentivirus carrying EGFP were used as negative control. (B) The overexpression of miR-181a in the K562 cells enhanced the inhibitory effect of the chemotherapeutic drug imatinib on the viability of these cells ( $\mathrm{P}<0.05$ vs. control cells). miR-181a, microRNA-181a; EGFP, enhanced green fluorescent protein; $\mathrm{IC}_{50}$, half maximal inhibitory concentration.

role of miR-181a in CML, miR-181a was overexpressed in the K562 cells and the consequent effects on the chemotherapeutic sensitivity of K562 cells to imatinib, the current first-line treatment for patients with CML in the clinic, were evaluated. qPCR analysis was used to measure the relative expression levels of miR-181a, with cells infected with EGFP as a control, and the results demonstrated that the expression
A
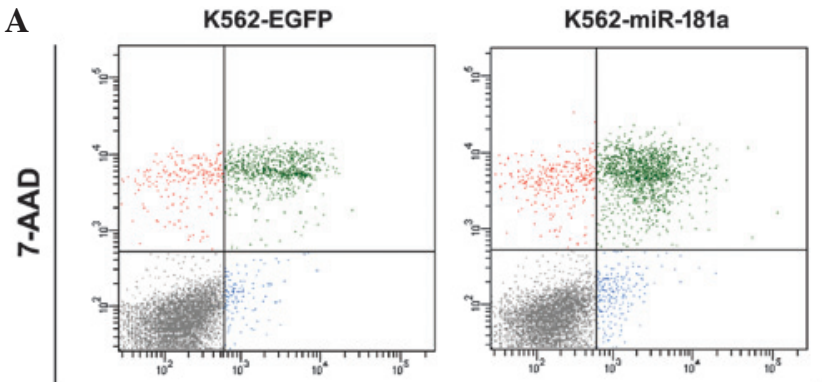

PE Annexin V

B

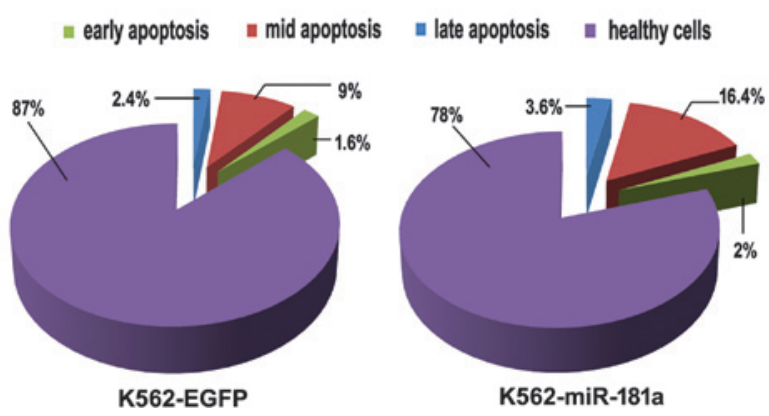

Figure 3. Overexpression of miR-181a in chronic myeloid leukemia K562 cells promotes the apoptosis of K562-miR-181a cells induced by the chemotherapeutic agent imatinib. (A) The apoptotic K562-miR-181a and K562-EGFP cells were stained with PE-Annexin V and 7-AAD, and detected by flow cytometry. (B) Graphical representation of the percentage of K562 cells undergoing apoptosis at different stages of the process (early, mid and late apoptosis), compared with the percentage of healthy cells. K562-EGFP cells were used as the control. miR-181a, microRNA-181a; 7-AAD, 7-aminoactinomycin D; PE, phycoerythrin; EGFP, enhanced green fluorescent protein.

A
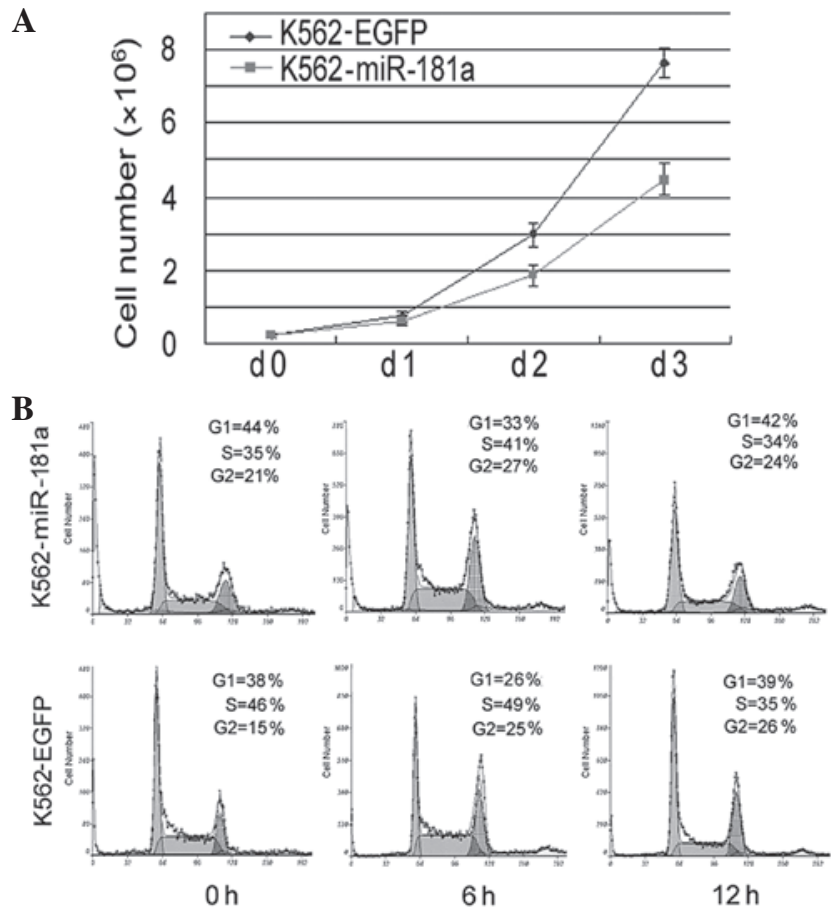

Figure 4. Overexpression of miR-181a in chronic myeloid leukemia K562 cells inhibits the proliferation of these cells by repressing the transition from the $G_{1}$ to $S$ phase of the cell cycle. (A) miR-181a inhibited the growth of the K562 cells overexpressing miR-181a compared with the control K562-EGFP cells. (B) miR-181a prevented the K562 cells from undergoing the $G_{1} / S$ phase transition of the cell cycle. Compared with the control cells, a larger number of K562-miR-181a cells were arrested at the $G_{1}$ phase of the cell cycle, whereas the opposite was observed for the number of cells arrested at the $S$ phase of the cell cycle. miR-181a, microRNA-181a; EGFP, enhanced green fluorescent protein. 
A

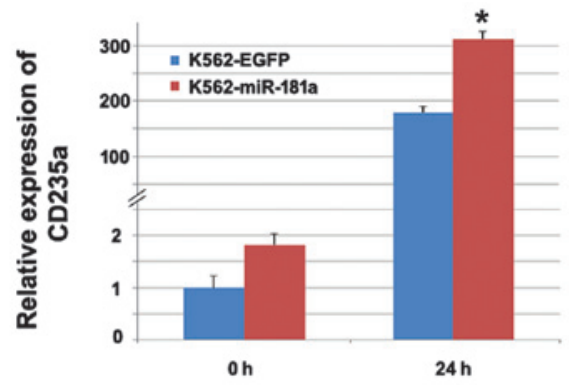

B

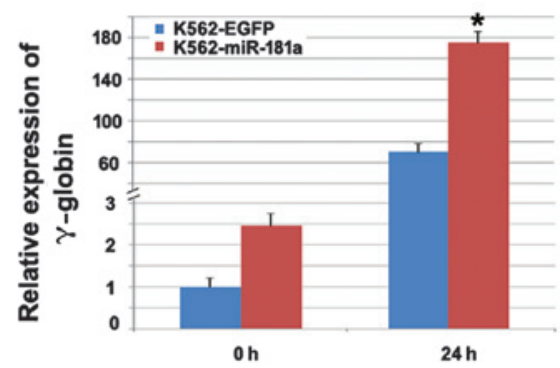

C

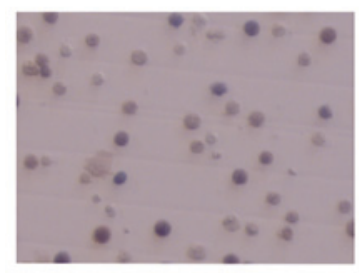

K562-EGFP

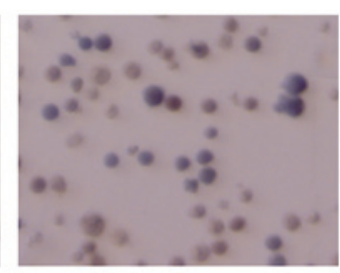

K562-miR-181a

D

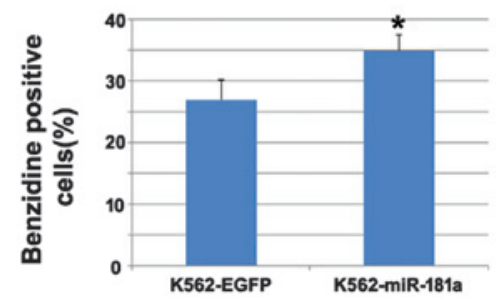

Figure 5. Overexpression of miR-181a promotes the erythroid differentiation of chronic myeloid leukemia K562 cells. The overexpression of miR-181a induced the expression of (A) CD235a and (B) $\gamma$-globin in the K562-miR-181a cells (" $\mathrm{P}<0.05$ vs. K562-EGFP control cells). (C) Benzidine staining confirmed the erythroid differentiation of K562-miR-181a cells (magnification, $\mathrm{x} 200$ ). (D) The overexpression of miR-181a in the K562 cells increased the number of benzidine-positive cells ("P<0.05 vs. K562-EGFP control cells). miR-181a, microRNA-181a; CD, cluster of differentiation; EGFP, enhanced green fluorescent protein.

levels of miR-181a were 8.3-fold higher in the K562 cells overexpressing miR-181a compared with the control cells $(\mathrm{P}<0.05$; Fig. 2A). Next, the K562 cells overexpressing miR-181a and the control cells were treated for $72 \mathrm{~h}$ with different concentrations of imatinib $(0.025,0.05,0.1,0.2,0.4,0.6$ or $0.8 \mu \mathrm{M})$, prior to be subjected to RT-qPCR analysis. The results revealed that the half maximal inhibitory concentration of imatinib in the K562-miR-181a cells was significantly lower than that in the control cells ( 0.23 vs. $0.29 \mu \mathrm{M}$, respectively; $\mathrm{P}<0.023$; Fig. $2 \mathrm{~B}$ ), which suggested that the overexpression of miR-181a in K562 cells enhances the sensitivity of these CML cells to imatinib.

miR-181a promotes the apoptosis of K562 cells induced by imatinib. To identify the potential mechanism mediating the increased sensitivity of K562 cells overexpressing miR-181a to the chemotherapeutic agent imatinib, the effect of
A

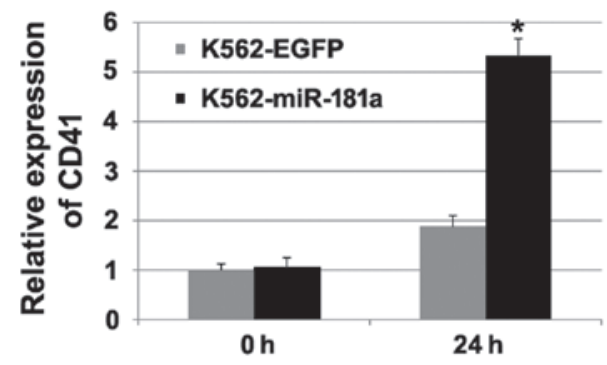

B

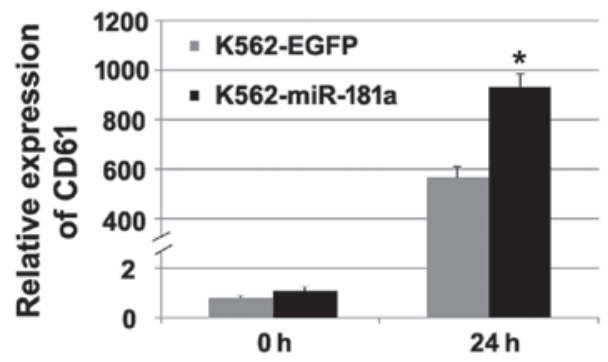

Figure 6. Overexpression of miR-181a promotes the megakaryocytic differentiation of chronic myeloid leukemia K562 cells. The overexpression of miR-181a induced the expression of (A) CD41 and (B) CD61 in the K562-miR-181a cells ( $\mathrm{P}<0.05$ vs. K562-EGFP control cells). miR-181a, microRNA-181a; CD, cluster of differentiation; EGFP, enhanced green fluorescent protein.

miR-181a on the apoptosis of the K562 cells was evaluated. Compared with the control cells, the apoptosis induced by imatinib was increased by $\sim 10 \%$ in the K562-miR-181a cells (Fig. 3A and B), which suggested that miR-181a promotes the apoptosis of K562 cells.

miR-181a inhibits the growth of K562 cells by repressing the $G_{I} / S$ phase transition of the cell cycle. The effect of miR-181a on the proliferation of the K562 cells was assessed via a cell growth curve assay. The results indicated that the overexpression of miR-181a significantly inhibited the proliferation of K562 cells (Fig. 4A). To evaluate the effect of miR-181a on the cell cycle, the number of K562-miR-181a cells that were arrested at the $\mathrm{G}_{1}$ and $\mathrm{S}$ phases of the cell cycle was measured at different time-points and compared with the number of K562-EGFP cells arrested at these phases. Pre-synchronization of the K562-miR-181a and K562-EGFP cells was achieved by serum starvation. The results demonstrated that the percentage of K562-miR-181a cells arrested at $\mathrm{G}_{1}$ was higher than the percentage of K562-EGFP cells, while the opposite was observed for the percentage of cells arrested at the $\mathrm{S}$ phase of the cell cycle (Fig. 4B). These findings suggested that the overexpression of miR-181a inhibits the $\mathrm{G}_{1} / \mathrm{S}$ phase transition of the cell cycle in K562 cells.

Overexpression of miR-181a promotes the differentiation of $\mathrm{K} 562$ cells. The differentiation status of leukemia cells usually affects the sensitivity of their response to chemotherapy treatment (15). In addition, the chemotherapeutic agents used for the treatment of leukemia may promote the differentiation of leukemia cells (16). Previous studies have demonstrated that bipotent K562 cells may be induced to undergo erythroid and megakaryocytic differentiation by hemin and TPA, respectively (10). In the present study, the 
effect of miR-181a on the erythroid and megakaryocytic differentiation of K562 cells was investigated.

The erythroid differentiation of the K562 cells was evaluated by benzidine staining and by the expression levels of CD235a and $\gamma$-globin, following induction with hemin. The results indicated that the overexpression of miR-181a in the K562 cells enhanced the expression of CD235a $(\mathrm{P}<0.05$; Fig. 5A) and $\gamma$-globin $(\mathrm{P}<0.05$; Fig. 5B), and increased the percentage of benzidine-positive cells, from $27 \%$ in the K562-EGFP cells to $36.5 \%$ in the K562-miR-181a cells $(\mathrm{P}<0.05$; Fig. 5C and $\mathrm{D})$. These findings suggested that miR-181a promotes the erythroid differentiation of K562 cells.

The megakaryocytic differentiation of the K562 cells was evaluated according to the expression levels of CD41 and CD61 following treatment with TPA. The results indicated that the overexpression of miR-181a promoted the expression of CD41 and CD61 ( $\mathrm{P}<0.05$ vs. control cells; Fig. 6A and B), which suggested that miR-181a promotes the megakaryocytic differentiation of K562 cells. Taken together, these results demonstrated that miR-181a promotes cell differentiation in CML K562 cells.

\section{Discussion}

Previous studies have suggested that miR-181 may act as a tumor suppressor or as an oncogene, depending on the cancer type. The downregulation of miR-181 has been observed to be critical for the development of B-CLL, and its loss has also been associated with adverse prognosis in patients with cytogenetically abnormal AML $(12,14)$. The downregulation of miR-181, due to the activation of nuclear factor $-\kappa \mathrm{B}$, has been reported to contribute to a poor clinical outcome in patients with estrogen receptor-positive breast tumors by enhancing stem cell-like properties in these tumor cells (17). Similarly, low expression levels of miR-181a have been associated with the poor differentiation of colorectal cancer (CRC) cells, and with decreased survival times of patients with CRC (18). In a model of human glioma, miR-181a and miR-181b induced cell apoptosis and inhibited cell proliferation and invasion (19). These results suggested that miR-181 is a tumor suppressor. However, in hepatic carcinoma, members of the miR-181 family were observed to be significantly upregulated, and the overexpression of miR-181 promoted hepatocarcinogenesis and the migration of cancer cells $(20,21)$. Furthermore, miR-181 was also observed to stimulate progression in breast cancer $(22,23)$. Therefore, these results indicate that miR-181 also acts as an oncogene in certain types of cancer. Therefore, the best of our knowledge, the present study investigated the function of miR-181a in CML for the first time.

Previous studies have demonstrated that miR-181 sensitizes malignant human glioma cells to radiation, which suggests that miR-181a may affect the sensitivity to chemotherapy treatment of certain types of cancer (24). To evaluate the effect of miR-181a in the treatment of CML, the effect of miR-181a on the chemotherapeutic sensitivity of CML K562 cells to imatinib was investigated, and the results indicated that the overexpression of miR-181a in the K562 cells increased the chemotherapeutic sensitivity of these cells to imatinib. These findings are in agreement with a previous study, which reported that miR-181c was downregulated in imatinib-resistant CML (25). In order to identify the potential mechanism by which miR-181a sensitizes
K562 cells to the chemotherapeutic effects of imatinib, the impact of miR-181a on the apoptosis and proliferation of K562 cells was evaluated. The results demonstrated that miR-181a promoted the apoptosis of K562 cells induced by imatinib and inhibited the proliferation of K562 cells by blocking the transition from the $G_{1}$ to $S$ phase of the cell cycle. Previous studies have reported that miR-181 targets B-cell lymphoma 2 (Bcl-2), an anti-apoptotic gene frequently overexpressed in cancer, which contributes to the cell apoptosis induced by miR-181 $(24,26)$. Therefore, targeting Bcl-2 may be an additional mechanism by which miR-181a promotes the imatinib-induced apoptosis of K562 cells.

miR-181 has been previously demonstrated to promote cell differentiation. miR-181 has been observed to facilitate the differentiation and development of immunity cells (including natural killer cells, and B and T lymphocytes), and to promote the differentiation of mammalian myoblasts, human embryonic stem cells and osteoblasts, by targeting different genes (3-8,27-29). Since the differentiation status of the tumor cells usually affects their sensitivity to chemotherapy, the effect of miR-181a on the differentiation of K562 cells was evaluated in the present study. The results indicated that the overexpression of miR-181a promoted the erythroid and megakaryocytic differentiation of the K562 cells, which may also contribute to the enhanced chemotherapeutic sensitivity exhibited by these cells towards the chemotherapeutic agent imatinib.

In summary, the present study demonstrated that the overexpression of miR-181a in CML K562 cells enhances the chemotherapeutic sensitivity of these cells to imatinib. These effects may be due to the capacity of miR-181a to inhibit cell growth, and/or to induce apoptosis and differentiation in CML cells.

\section{Acknowledgements}

The present study was partially supported by the National Natural Science Foundation of China (grants nos. 81401961 and 81302061), the Postdoctoral Scientific Research Development Fund of Heilongjiang Province (grant no. LBH-Q14104) the Wu-Lian-De Youth Science Foundation of Harbin Medical University (grant no. WLD-QN1411), the Science and Technology Plan Foundation of Inner Mongolia Autonomous Region (grant no. 20130404) and the Hospital Foundation of Inner Mongolia Autonomous Region People's Hospital (grant no. 201301).

\section{References}

1. Ambros V: MicroRNAs: Tiny regulators with great potential. Cell 107: 823-826, 2001.

2. Guarnieri DJ and DiLeone RJ: MicroRNAs: A new class of gene regulators. Ann Med 40: 197-208, 2008.

3. Chen CZ, Li L, Lodish HF and Bartel DP: MicroRNAs modulate hematopoietic lineage differentiation. Science 303: 83-86, 2004.

4. Cichocki F, Felices M, McCullar V, Presnell SR, Al-Attar A, Lutz CT and Miller JS: Cutting edge: MicroRNA-181 promotes human NK cell development by regulating Notch signaling. J Immunol 187: 6171-6175, 2011.

5. Ziętara N, Łyszkiewicz M, Witzlau K, Naumann R, Hurwitz R, Langemeier J, Bohne J, Sandrock I, Ballmaier M, Weiss S, et al: Critical role for miR-181a/b-1 in agonist selection of invariant natural killer T cells. Proc Natl Acad Sci USA 110: 7407-7412, 2013 
6. Henao-Mejia J, Williams A, Goff LA, Staron M, Licona-Limón P, Kaech SM, Nakayama M, Rinn JL and Flavell RA: The microRNA miR-181 is a critical cellular metabolic rheostat essential for NKT cell ontogenesis and lymphocyte development and homeostasis. Immunity 38: 984-997, 2013.

7. Li QJ, Chau J, Ebert PJ, Sylvester G, Min H, Liu G, Braich R, Manoharan M, Soutschek J, Skare P, et al: miR-181a is an intrinsic modulator of T cell sensitivity and selection. Cell 129: 147-161, 2007.

8. Belkaya S and van Oers NS: Transgenic expression of microRNA-181d augments the stress-sensitivity of CD4(+) CD8(+) thymocytes. PLoS One 9: e85274, 2014.

9. Choong ML, Yang HH and McNiece I: MicroRNA expression profiling during human cord blood-derived CD34 cell erythropoiesis. Exp Hematol 35: 551-564, 2007.

10. Li X, Zhang J, Gao L, McClellan S, Finan MA, Butler TW, Owen LB, Piazza GA and Xi Y: MiR-181 mediates cell differentiation by interrupting the Lin 28 and let-7 feedback circuit. Cell Death Differ 19: 378-386, 2012.

11. Pekarsky Y, Santanam U, Cimmino A, Palamarchuk A, Efanov A, Maximov V, Volinia S, Alder H, Liu CG, Rassenti L, et al: Tcl1 expression in chronic lymphocytic leukemia is regulated by miR-29 and miR-181. Cancer Res 66: 11590-11593, 2006.

12. Calin GA, Pekarsky Y and Croce CM: The role of microRNA and other non-coding RNA in the pathogenesis of chronic lymphocytic leukemia. Best Pract Res Clin Haematol 20: 425-437, 2007.

13. Marcucci G, Maharry K, Radmacher MD, Mrózek K, Vukosavljevic T, Paschka P, Whitman SP, Langer C, Baldus CD, Liu CG, et al: Prognostic significance of, and gene and microRNA expression signatures associated with, CEBPA mutations in cytogenetically normal acute myeloid leukemia with high-risk molecular features: A Cancer and Leukemia Group B Study. J Clin Oncol 26: 5078-5087, 2008.

14. Li Z, Huang H, Li Y, Jiang X, Chen P, Arnovitz S, Radmacher MD, Maharry K, Elkahloun A, Yang X, et al: Up-regulation of a HOXA-PBX3 homeobox-gene signature following down-regulation of miR-181 is associated with adverse prognosis in patients with cytogenetically abnormal AML. Blood 119: 2314-2324, 2012.

15. Pan XN, Chen JJ, Wang LX, Xiao RZ, Liu LL, Fang ZG, Liu Q, Long ZJ and Lin DJ: Inhibition of c-Myc overcomes cytotoxic drug resistance in acute myeloid leukemia cells by promoting differentiation. PLoS One 9: e105381, 2014.

16. Yang Y, Liu X, Xiao F, Xue S, Xu Q, Yin Y, Sun H, Xu J, Wang H, Zhang Q, Wang $\mathrm{H}$ and Wang L: Spred2 modulates the erythroid differentiation induced by imatinib in chronic myeloid leukemia cells. PLoS One 10: e0117573, 2015.

17. Kastrati I, Canestrari E and Frasor J: PHLDA1 expression is controlled by an estrogen receptor-NFKB-miR-181 regulatory loop and is essential for formation of $\mathrm{ER}^{+}$mammospheres. Oncogene 34: 2309-2316, 2015.
18. Pichler M, Winter E, Ress AL, Bauernhofer T, Gerger A, Kiesslich T, Lax S, Samonigg H and Hoefler G: miR-181a is associated with poor clinical outcome in patients with colorectal cancer treated with EGFR inhibitor. J Clin Pathol 67 198-203, 2014.

19. Shi L, Cheng Z, Zhang J, Li R, Zhao P, Fu Z and You Y: hsa-miR-181a and hsa-miR-181b function as tumor suppressors in human glioma cells. Brain Res 1236: 185-193, 2008.

20. Wang B, Hsu SH, Majumder S, Kutay H, Huang W, Jacob ST and Ghoshal K: TGF $\beta$-mediated upregulation of hepatic miR-181b promotes hepatocarcinogenesis by targeting TIMP3. Oncogene 29: 1787-1797, 2010.

21. Song MK, Park YK and Ryu JC: Polycyclic aromatic hydrocarbon (PAH)-mediated upregulation of hepatic microRNA-181 family promotes cancer cell migration by targeting MAPK phosphatase-5, regulating the activation of p38 MAPK. Toxicol Appl Pharmacol 273: 130-139, 2013.

22. Neel JC and Lebrun JJ: Activin and TGF $\beta$ regulate expression of the microRNA-181 family to promote cell migration and invasion in breast cancer cells. Cell Signal 25: 1556-1566, 2013.

23. Mansueto G, Forzati F, Ferraro A, Pallante P, Bianco M, Esposito F, Iaccarino A, Troncone $G$ and Fusco A: Identification of a new pathway for tumor progression: MicroRNA-181b up-regulation and CBX7 down-regulation by HMGA1 protein. Genes Cancer 1: 210-224, 2010.

24. Chen G, Zhu W, Shi D, Lv L, Zhang C, Liu P and Hu W: MicroRNA-181a sensitizes human malignant glioma U87MG cells to radiation by targeting Bcl-2. Oncol Rep 23: 997-1003, 2010.

25. Mosakhani N, Mustjoki S and Knuutila S: Down-regulation of miR-181c in imatinib-resistant chronic myeloid leukemia. Mol Cytogenet 6: 27, 2013.

26. Ouyang YB, Lu Y, Yue S and Giffard RG: miR-181 targets multiple Bcl-2 family members and influences apoptosis and mitochondrial function in astrocytes. Mitochondrion 12: 213-219, 2012.

27. Naguibneva I, Ameyar-Zazoua M, Polesskaya A, Ait-Si-Ali S, Groisman R, Souidi M, Cuvellier S and Harel-Bellan A: The microRNA miR-181 targets the homeobox protein Hox-A11 during mammalian myoblast differentiation. Nat Cell Biol 8: 278-284, 2006

28. Xu Z, Jiang J, Xu C, Wang Y, Sun L, Guo X and Liu H: MicroRNA-181 regulates CARM1 and histone arginine methylation to promote differentiation of human embryonic stem cells. PLoS One 8: e53146, 2013.

29. Bhushan R, Grünhagen J, Becker J, Robinson PN, Ott CE and Knaus P: miR-181a promotes osteoblastic differentiation through repression of TGF- $\beta$ signaling molecules. Int J Biochem Cell Biol 45: 696-705, 2013. 